\title{
Tracking a Single lon in an Ultracold Gas
}

\author{
Direct observation of an ion moving through a Bose-Einstein condensate \\ identifies the effect of ion-atom collisions on charge transport in an \\ ultracold gas.
}

\author{
By Johannes Hecker Denschlag
}

W hen you expose mobile electrical charges in a medium to an electrical field, current flows. The charges are accelerated by the field, but collisions within the medium give rise to a kind of friction effect, which limits the velocity of the charges and thus the current. This universal concept, called diffusive transport, describes a large range of media, such as metallic conductors, electrolytic solutions, and gaseous plasmas. But in a quantum system, such as a superconductor or a superfluid, other collective effects can influence the transport through the medium. Now, a group led by Florian Meinert and Tilman Pfau both of the University of Stuttgart, Germany, have carried out charge-transport

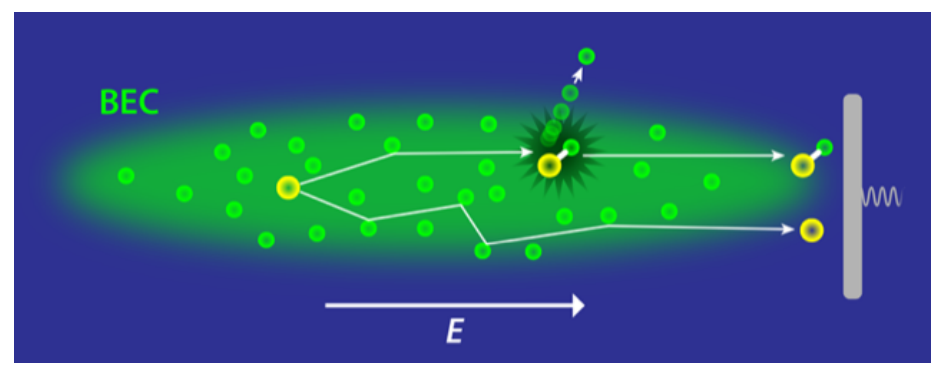

Figure 1: Researchers have designed an experiment that generates a single ion (yellow) inside a Bose-Einstein condensate (BEC) of ultracold rubidium atoms (green). The ion is accelerated by an electric field, and its transport distance is measured with a detector system (shown on the right). The data show that the ion moves diffusely through the BEC. The observations are also able to characterize chemical reactions that occur between the ion and the atoms.

Credit: Credit: APS/Alan Stonebraker experiments with a single ion traversing a Bose-Einstein condensate (BEC), which is a quantum gas of cold neutral atoms [1]. The precise tracking of the ion shows that the transport is diffusive and reveals the character of the ion-atom collisions. The results pave the way for future work that should be able to uncover the presence of quantum effects that influence transport.

Researchers have been unsure about what exactly to expect when an ion is immersed in a BEC. Since the BEC is a superfluid, one might assume that transport will be frictionless and, therefore, not diffusive. On the other hand, some theorists have hypothesized that a large cluster of the atoms might form around the ion, which should impede charge transport and produce diffusive signatures [2]. But other quantum effects might also play a role. Resonant charge transfer between the ion and a neighboring atom could lead to a drastic increase of the conductivity in the gas because, in this case, the charge transport is based on the motion of the light electron rather than that of the heavy ion [3].

To investigate the transport scenario experimentally, the Stuttgart-based group studied a single rubidium ion $\left(\mathrm{Rb}^{+}\right)$in a cigar-shaped $B E C$ of about one million neutral rubidium $(\mathrm{Rb})$ atoms [1]. The team initially produced the $\mathrm{Rb}^{+}$ion in the center of the $50-\mu \mathrm{m}$-long Rb cloud through a two-step process. First, they focused a laser beam at the cloud center, causing a single $\mathrm{Rb}$ atom to be excited to a Rydberg state, characterized by an electron occupying an orbital with a large effective radius. Only one atom was excited in this way, as strong interactions with this Rydberg atom shifted the energy levels of its neighbors so 
that they were out of resonance with the laser frequency-an effect called Rydberg blockade [4]. Afterwards, the Rydberg atom was ionized by applying a small dc electric field pulse. The process produced ${\mathrm{a} \mathrm{Rb}^{+}}^{+}$ion with a small initial velocity of about $10 \mathrm{~cm} / \mathrm{s}$, which corresponds to a temperature of $50 \mu \mathrm{K}$.

Next, the researchers carried out charge-transport experiments by applying a tunable, homogenous electric field of a few $\mathrm{mV} / \mathrm{cm}$, which accelerated the cold ion along the long-axis direction of the BEC. After a chosen "transport" time of up to 25 $\mu \mathrm{s}$, the team measured the location of the ion with micrometer resolution by turning on a strong electric field that accelerated the ion toward a multichannel plate ion detector. From the time delay between the field switch-on and the detection click on the multichannel plate, they could infer where the ion was located at the end of the transport phase.

The experiments show that the ion moves a shorter distance in a dense cloud than in a dilute one. This reduction can be explained by the collisions of the ion with the ultracold atoms, which slow down the ion. For the given particle density of $4 \times 10^{14} \mathrm{~cm}^{-3}$, collisions take place about once every microsecond. A constant drift velocity of the ion, where acceleration and friction are balanced, is expected to be established after a few collisions. However, because the atomic cloud is quite small, this regime is reached just briefly before the ion leaves the atomic cloud. Nevertheless, the data allow the researchers to conclude that the drift velocity increases linearly with the applied electric field, as expected for standard diffusive transport of the atomic ion.

The results show the role of "classical" collisions on charge transport, but questions remain over what other effects might be present. The data cannot yet reveal whether atom clusters form around the ion or if electron hopping occurs between the ion and other atoms. The BEC's superfluidity should suppress collisions, but to get into the right regime, experiments may have to be performed with even colder ions and lower electrical acceleration fields.

The current experimental setup does, however, offer another window into ion behavior, besides transport. The researchers observe that a large fraction of the $\mathrm{Rb}^{+}$ions undergo a particular chemical reaction, three-body recombination, with $\mathrm{Rb}$ atoms $[5,6]$. Here, an ion forms a weakly bound $\mathrm{Rb}_{2}^{+}$dimer with a $\mathrm{Rb}$ atom, while a second $\mathrm{Rb}$ atom carries away part of the released binding energy. The $\mathrm{Rb}_{2}^{+}$dimer can be easily discriminated from the $\mathrm{Rb}^{+}$ion because it arrives later at the ion detector as a result of its larger mass.

Remarkably, the group was able to estimate the typical binding energy of the $\mathrm{Rb}_{2}^{+}$dimer in a separate set of experimental runs [5]. By applying a suitable electric field, the weakly bound $\mathrm{Rb}_{2}^{+}$ dimer can be ripped apart-converting it back to $\mathrm{Rb}^{+}$ion and a neutral $\mathrm{Rb}$ atom. The larger the binding energy, the stronger the electric field needed to dissociate the molecule. Thus, by varying the electrical field and measuring the percentage of dimers that were dissociated, the team determine their binding energies.

The binding-energy observations reveal that the molecular ion changes as it traverses the cloud: it becomes more and more deeply bound after its production. This relaxing to lower energy states is due to inelastic secondary collisions of the $\mathrm{Rb}_{2}^{+}$ molecule with $\mathrm{Rb}$ atoms. Similar relaxation dynamics have been recently observed by our group in Ulm, Germany, with a $\mathrm{BaRb}^{+}$molecule, where we employed a different optical detection method for the molecules [7]. These experiments can offer a way to probe chemical reaction channels on the quantum level. This so-called state-to-state chemistry will require resolving the quantized molecular energy levels-a capability that seems within reach for the Stuttgart group.

The new ion-atom experiments by Meinert, Pfau, and colleagues provide an important verification of diffusive transport in a BEC. It will be very exciting if the measurements can be refined to also find evidence for the formation of the predicted atomic clusters around the ion [2], for fast charge transport due to charge hopping between the particles [3], or for frictionless transport of the ion in a BEC. If their setup can be optimized to resolve quantized molecular energy levels, interesting experiments on state-to-state chemistry should be possible.

Johannes Hecker Denschlag: Institute of Quantum Matter, Ulm University, Ulm, Germany

\section{REFERENCES}

1. T. Dieterle et al., "Transport of a single cold ion immersed in a Bose-Einstein condensate," Phys. Rev. Lett. 126, 033401 (2021). 
2. R. Côté et al., "Mesoscopic molecular ions in Bose-Einstein condensates," Phys. Rev. Lett. 89, 093001 (2002).

3. R. Côté, "From classical mobility to hopping conductivity: Charge hopping in an ultracold gas," Phys. Rev. Lett. 85, 5316 (2000).

4. E. Urban et al., "Observation of Rydberg blockade between two atoms," Nat. Phys. 5, 110 (2009).
5. T. Dieterle et al., "Inelastic collision dynamics of a single cold ion immersed in a Bose-Einstein condensate," Phys. Rev. A 102, 041301 (2020).

6. A. Härter et al., "Single ion as a three-body reaction center in an ultracold atomic gas," Phys. Rev. Lett. 109, 123201 (2012).

7. A. Mohammadi et al., "Life and death of a cold $\mathrm{BaRb}^{+}$molecule inside an ultracold cloud of Rb atoms," arXiv:2005.09338. 